\title{
Fragmented responsibility: views of Israeli HCPs regarding patient recontact following variant reclassification
}

\author{
Alma Levin Fridman ${ }^{1} \cdot$ Aviad Raz $^{1} \cdot$ Stefan Timmermans ${ }^{2} \cdot$ Shiri Shkedi-Rafid $^{3}$ \\ Received: 26 August 2021 / Accepted: 27 September 2021 / Published online: 5 October 2021 \\ (c) The Author(s), under exclusive licence to Springer-Verlag GmbH Germany, part of Springer Nature 2021
}

\begin{abstract}
While genomic medicine is becoming an important part of patient care with an ever-increasing diagnostic yield, recontacting patients after reclassification of variants of uncertain clinical significance (VUSs) remains a major challenge. Although periodical reinterpretation of VUSs is highly desired, recontacting former patients with new classifications is commonly not fulfilled in practice. We draw on semi-structured interviews with 20 Israeli healthcare professionals and stakeholders involved in communicating the results of genome-wide sequencing to patients. Findings show agreement that an individual health care professional cannot address the task of recontacting patients after re-classification, and that responsibility should be shared among the medical specialties, laboratory scientists, as well as patients. In the absence of established guidelines, many respondents suggested that the patient should be informed about reclassification during a follow-up contact but they disagreed who should be responsible for informing the patient. HCPs agreed that the solution to this challenge involves a centralized automated database that is accessible, continuously updated, and facilitates retrospective as well as prospective flagging of reclassification for patients who can benefit from this information. National and international policies providing concrete guidelines on the optimal way to recontact patients with new valuable genomic information are needed.
\end{abstract}

Keywords VUSs $\cdot$ Reclassification $\cdot$ Patient recontact $\cdot$ WGS $\cdot$ Responsibility

\section{Introduction}

Genomic medicine imposes new opportunities and pressures for health care providers to recontact patients to provide updated interpretations of genomic information. With rapid advances in genomic technologies, whole-genome sequencing technologies are becoming faster and cheaper, hence available to a growing number of people. As a result, previously discovered variants of unknown/uncertain significance (VUSs) may be reclassified as pathogenic or non-pathogenic. Recent research suggests that about $8 \%$ of variants initially classified as VUSs were later either downgraded to less severe classification (about $91 \%$ of variants) or upgraded

Aviad Raz

aviadraz@bgu.ac.il

1 Department of Sociology and Anthropology, Ben-Gurion University of the Negev, Beersheba, Israel

2 Department of Sociology-UCLA, Los Angeles, CA, USA

3 Department of Genetics, Hadassah Medical Organization and Faculty of Medicine, Hebrew University of Jerusalem, Jerusalem, Israel to more severe classifications (about 9\% of variants; Mersch et al 2018). Thus, periodical reinterpretation of VUS is inevitable and may have significant implications for patients and their family members. However, re-classification involves multiple stakeholders: public as well as private genetics labs, physicians, genetic health professionals, and medical geneticists. In addition, molecular information is rarely transparently conclusive, but requires interpretation along with other sources of clinical and non-clinical information. While multidisciplinary collaborations are considered in the literature as one of the most effective ways to reduce misunderstandings about roles and responsibilities between healthcare professionals in the management of patients, such collaborations can also be hard to implement (Carrieri et al. 2017; Doheny et al. 2018).

There is yet no consensus and no standard policy over whether, when, who exactly, and how patients affected by such re-classification should be recontacted. Professional organizations such as the American College of Medical Geneticists (ACMG) generally advise that re-contact is fundamentally a shared responsibility between the ordering health-care provider, the clinical testing laboratory, and the 
patients themselves (Richards et al. 2015; David et al. 2019). Similarly, the European Society of Human Genetics (ESHG) recommended that recontacting patients in clinical genetics services should be commensurate with previously obtained consent, and a shared responsibility between healthcare providers, laboratories, patients (who may be asked to take the initiative to check in), and other stakeholders (Carrieri et al. 2019; Doheny et al. 2018). The only systematic review of the literature (Otten et al. 2015) on recontacting in clinical genetics highlights recontacting as ethically desirable yet neither fulfilled in practice nor logistically feasible.

In recent years, an increasing number of studies (Medendorp et al. 2020, 2021) suggest that with greater powers of reclassification come greater challenges of recontact. The general recommendations of the ACMG and the ESHG leave open a series of practical questions. When new information that sheds light on the clinical significance of a variant is discovered, is it the responsibility of the laboratory, the clinician, or the genetic counselor to contact the person who carries the mutation? Or is it the responsibility of the patient to periodically check back? Where does responsibility lie, and what kind of evidence should trigger a need to share newfound information? (Shabani et al. 2016; David et al. 2019; Appelbaum et al. 2020).

Importantly, while these tasks are perceived as ethical duties for HCPs, their legal implications are not well defined (Clayton et al. 2021). While no courts, as far as we know, have yet imposed liability for failure to recontact patients with new genetic results, the challenges of ensuring that patients receive and understand the new results loom large. ${ }^{1}$ A recent UK survey showed that genetics services do recontact former patients but in an ad hoc fashion. Furthermore, HCPs express uncertainty about who, when, and how should patients be recontacted following reclassification of VUSs (Carrieri et al. 2016; Doheny et al. 2018).

To provide much needed empirical evidence, this paper draws on interviews with Israeli healthcare professionals from clinical genetics, professionals from mainstream healthcare specialties, and legal experts. These were chosen as they are involved in "precision"/ "personalized" medicine either as clinicians refereeing to testing, interpreting genomic results, following up patients, or taking part in building guidelines. This study provides an in-depth investigation of their perspectives on the clinical, ethical, and legal issues related to recontacting.

\footnotetext{
$\overline{1}$ In Williams v. Quest Diagn., Inc., (423 S.C. 547 (S.C. 2018), plaintiff mother alleged that in 2007 Quest subsidiary of Athena Diagnostics erred when it classified as a VUS a variant in the SCN1A gene in her 2-year-old son (who died in 2008). The testing lab issued a revised report in 2015 re-categorizing the VUS as a pathogenic mutation. This is not a case of failed recontact but of retrospective reclassification. South Carolina Supreme Court ruled in Quest's favor.
}

\section{Methods}

We conducted semi-structured interviews as part of a broader study to investigate ethical and social issues of negotiating genome-wide sequencing (GWS) in clinical care. The sample was comprised of healthcare professionals who communicate GWS results to patients: 8 genetics counselors (two of them with cardio-genetic expertise), 5 medical geneticists (3 specialized in cardio-genetics), 5 oncologists, as well as 2 legal experts with bioethical expertise concerning GWS in Israel (total $n=20$ ). All HCPs had more than 5 years of experience in their current position.

Based on relevant literature and the clinical experience of the fourth co-author, the research team prepared the guide for the semi-structured interviews including questions addressing the following topics: (a) how medical geneticists, genetic counselors, and clinicians outside of genetics work together to decide what genetic information requires re-contact; and (b) how information about reclassified VUSs is reported and communicated with patients. Specific questions addressed experiences of recontacting and views about potential responsibilities for recontacting and the implementation of policies and systems to enable this. Following IRB approval, we contacted relevant (mostly onco-genetic) physicians and genetics counselors from 6 different medical centers across Israel. All interviews were conducted in Hebrew, audio recorded, transcribed, and thematized. Participants were identified and invited to participate by the clinical authors of this paper based on their professional experience and networks. We sampled purposefully: targeting HCPs who participated in writing professional guidelines concerning GWS. Interviews lasted between 30 and $60 \mathrm{~min}$, and, given the Covid-19 pandemic circumstances, were mostly conducted via telephone.

All those approached agreed to take part in the research. Interview transcripts were translated by the authors from Hebrew to English and pseudonyms were used with quotes. The transcripts were analyzed thematically to uncover discursive themes and categories of themes recurring within and across groups of respondents, for example groups of different HCPs (Denzin \& Lincoln 1994). Following a review of the relevant literature, preliminary codes included communication strategies, consent, and responsibility to recontact. Additional themes were inductively gleaned from the transcripts. The research team did the coding together on the first few interview transcripts, discussing the relevance of the themes and agreeing on needed modifications and reclassifications. The authors then continued with the coding, discussing new findings as they appeared and their relationships to the codes in team meetings, where agreements were reached to prevent the potential bias of a single rater, and using inter-rater reliability to increase the validity of 
the results. The quotes used were selected to represent the range of opinions among the respondents. We focus here on views presented concerning the responsibility to recontact following VUS reclassification.

\section{Results}

\section{The organizational challenge of reclassification and recontact}

Respondents saw reclassification leading to recontact as a complex and concerted multi-disciplinary endeavor that lacks proper organization. Respondents agreed that one cannot address this task alone, and the responsibility should be shared among all the medical specialties and laboratory scientists involved in the diagnosis, treatment, and management of patients. However, this consensus among respondents was often the basis for dissatisfaction, criticism, and recognition of personal and systemic constraints.

"I try to keep updated, I go to conferences, I read the ACMG guidelines [...] but do I really have the time to read papers [about reclassification] that often? $(\mathrm{F}$, genetic counselor)

It's over my head, it's not practical that a physician remains updated about every possible VUS and every possible mutation, it's not my job." (C, oncologist)

Many respondents stressed the need for a centralized web of data processing where information on VUS reclassification can be updated and shared:

"We need a central genomic database otherwise everything goes to the trash bin. Data gets stuck in an email to the doctor or in some report. Otherwise, when some VUS is reclassified, nobody will tell you [...] In an ideal world everything would have been digitized and there would be a central genomic database that is constantly being updated." (A, oncologist)

Many respondents explained that reclassification is going to happen in future cases, but unfortunately too often it is not applied to retrospective cases of patients that could have benefitted from it:

"If a report was issued some years ago on some mutation that was classified as VUS, and today we know it is not a VUS, those that issued the report do not go back to correct the report that is still circulating. Only future reports [by the testing lab] will have the correct information. It implies potential and actual errors for real patients." (C, oncologist)

"In an ideal world, we would have a system where we upload the variants [...] after one year, the sys- tem would alert us to check for reclassification [...] in an even better world the system would check for the reclassification independently." (A, genetics counselor)

\section{Lack of guidelines and routines concerning recontact}

All our respondents stressed the current lack of any national regulations to guide recontacting:

"There are no guidelines or regulations even though it has been discussed for several years but still there is nothing." (R, legal expert)

Most respondents found the lack of guidelines and regulation worrisome given the potential clinical and legal consequences of (not) recontacting. A minority view, however, emphasized that this lack of regulation also has benefits:

"It is a very dynamic field and sometimes you must let the field have enough leeway to decide on its own. Currently it depends on the genetics service- a patient with the same VUS might receive information about that VUS and its reclassification in one genetics institute but not in another." (S, legal expert)

Other respondents wished for general rules of thumb that would still leave them with leeway for professional consideration:

"There should be some rules concerning what requires obligatory recontact, rather than regulating each specific case $[. .$.$] probably the obligation should be to$ recontact about reclassification to pathogenic or likely pathogenic, as well as reclassification that concerns the ACMG gene list [to be reported as secondary findings]. This is the minimum, and it leaves room for consideration." (I, geneticist)

The genetic HCPs reported that they recontact patients but only occasionally, for example, if a new family member is referred to the clinic and this triggers a review of the family files (especially children of parents with a genetic condition that considers pregnancy), or in cases they find out about actionable reclassifications of VUSs. However, there was no routine for recontacting, and the cases described were the result of ad-hoc circumstances and often had to rely on the HCPs' memory and personal familiarity with the case. Accessible and continuously updated databases, or their lack thereof, were often mentioned as a necessary first step for effective recontacting policies.

\section{Whose responsibility?}

Respondents expressed a variety of views regarding whose responsibility it is to initiate recontact concerning VUS 
reclassification. Many respondents argued in favor of the idea that patients should share responsibility for recontacting by agreeing to get in touch with healthcare professionals at regular intervals to ask for updates. This was presented as being advisable with current limited resources, and all the respondents agreed that they cannot initiate recontact systematically.

"You come to recognize it when a patient comes back to you for consultation, and you open their file and you ask again. I would not do it for someone I haven't seen for a year. I think we should tell patients, also for medico-legal reasons, please come back in a year or so and nudge us." (M, genetics counselor)

However, respondents also acknowledged that transferring the responsibility to the patient is not always appropriate given that patients are diverse:

"Some patients come back. Some patients are very disciplined, and they want to schedule a meeting two years ahead. But for sure many other patients do not follow up and will never come back." (M, genetics counselor)

Nevertheless, the majority view among our respondents was that it is legitimate to share responsibility with patients:

"It is legitimate, they are grown-up people. Every clinician can tell their patient, see me again in one year. If a patient is irresponsible and doesn't come back, it's on them. Our responsibility is to explain why it is important. I would not feel bad with myself if I did that and the patient did not follow-up." (F, genetics counselor) "Our current policy in the Genetics Institute is that the responsibility is on the patient, although at some point we would probably have to develop some automated mechanism for reclassification every year or so. So far, we have avoided that responsibility. We are certainly not obligated to do it." (H, senior geneticist)

A minority view stressed that responsibility should be shared between the patient, the HCP, and the laboratory.

Patients should take note that in half a year they call the genetics service and do follow-up. You also have responsibility, it's not a one-off deal, and it's not our job to chase you around. When it comes to VUS it's your responsibility too. Is this responsibility split fiftyfifty, or maybe they have forty-nine and I have fiftyone? Probably I have fifty-one since in cases of reclassification to likely pathogenic my job is to recontact, that's on me." (G., senior geneticist)

Another minority opinion, expressed by a few of the oncologists, argued that the responsibility ultimately belongs to the genetic counselors.
"I think that's a tough question but ideally it would be the responsibility of the genetic counselors. It's difficult to follow-up on this and they would need a digitized database." (S, oncologist)

On the other hand, a few genetics counselors argued that because genetic counselling is done on an episodic basis, the patients and their physician should have the responsibility:

"We don't have the capacity to follow-up on the patients. The patient sees us for the consultation and then they don't see us anymore $[. .$.$] the patient should$ know he has to follow-up, and the physician who sees the patient every half a year can also assume responsibility for this.” (W, genetic counselor)

Importantly, all HCPs agreed that in practice, patients rarely come back for follow-ups. As one of the counselors typically remarked:

"Most patients don't come back to you [for follow-up].

But for those who do come back, of course I'll do the re-check thoroughly and give them complete assessment." (S, genetics counselor)

Finally, another physician argued that the lab should be responsible:

In the hospital, the lab should be responsible because they keep in touch with patients. If the patient doesn't continue to see me, then I don't have any follow-up on that patient. The lab should develop an algorithm that automatically updates the VUSs and raises flags. But anyhow it doesn't look reasonable to relay this responsibility to patients. It's too much to tell the patient: look, you have all these things that nobody knows what they mean, once a year you should follow-up with us to check if it didn't become important." (C, oncologist)

\section{Discussion}

Our findings highlight the various stakeholders involved in VUS reclassification and recontact: labs, clinicians, genetics health professionals, the genetics institute as an information and organizational hub, and patients. Responsibility is not well-defined regarding who is following up on reclassification and who initiates patient recontact after revision of genomic test results. The major theme found in the data was that no one considered themselves responsible for recontacting patients but thought that other parties should take the initiative. Many of the different stakeholders practically threw the responsibility back and forth among themselves. Major challenges that were mentioned included lack of guidelines, ambiguity about the legal and regulatory requirements, and, beyond that, the absence of a centralized database or 
an information infrastructure. The patient was seen as the most suitable stakeholder to ask to follow up on reclassification-a request which is nonetheless quite problematic since research has shown that patients are not always clear about the meaning of VUS results, or even that the clinician communicated such results (Reuter et al. 2019).

HCPs in other studies described the transfer of responsibility to the patient as an option which makes them uncomfortable, since patients may have little appreciation of the potential gravity of this task, or conversely find it a great burden, or even forget about the test (Doheny et al. 2018). Furthermore, the suggestion that recontacting could be a "joint venture" between patients and HCPs (Dheensa et al. 2017) has been criticized as having the potential to introduce inequity and to maintain or exacerbate disparities in health and health care (Carrieri et al. 2019). Those who are more burdened by disease, disability, access, and life contingencies, may be less likely to rise to this challenge, thus unfortunately reinforcing Tudor Hart's (1971) Inverse Care Lawthat the availability of good medical or social care tends to vary inversely with the need of the population served.

The challenges of reclassification and recontact are an indication of the relatively new and dynamic nature of GWS. HCPs have some responsibility to link reclassification and recontact, but they do not have the power to manage all the new data accumulated through GWS. Thus, fragmented responsibility may ultimately amount to a situation of organized irresponsibility (Braun and Könninger 2018). As other studies have shown, developments in sequencing technologies and research discourse that "suggest a need to recontact do not translate directly into the incorporation of a duty to recontact for clinicians" (Doheny et al. 2018: 188). The question becomes how actors involved with governing reclassification and recontact conceive their involvement and the responsibility that comes with it and how they implement this responsibility. Our respondents illustrate how stakeholder accounts of such realization do not necessarily precede practices but rather emerge as part of social and organizational interactions that characterize the challenge of translating genomics into "personalized" clinical care (Marchant et al. 2020).

The need to establish efficient protocols for recontacting is expected to increase with the proliferation of VUS and their reclassification, requiring a uniform standard of care. The sparse guidelines on communicating VUS that were created recently in the Israeli health system relate mainly to prenatal genetic diagnosis(Millo et al. 2021), but there is no mention of recontacting (Koifman et al. 2016). Our findings recapitulate and extend those of Carrieri et al. $(2017,2019)$ concerning the lack of, and need for, clear lines of responsibility for initiating recontact. As in other health systems, this is complicated by the increasing use of genetic testing by mainstream medical specialties. Genetic HCPs are often seen as preferred recontact agents given their ability to communicate complexities of genomic information and because of access to patient registers. However, as specialists, genetic HCPs see patients on an irregular basis and may lack the time and resources to handle reclassification and recontacts systematically without the existence of a dedicated central information infrastructure.

As many of our respondents suggested, there is an urgent need for automated databases that are accessible, are continuously updated, and facilitate retrospective as well as prospective flagging of reclassification for patients who can benefit from this information. Even if such systems are used, questions remain about the flow of information from the lab to the patient-who is best placed to contact the patient and what happens if $\mathrm{s} / \mathrm{he}$ is not accessible or deceased. Patient apps, such as GenomeConnect (the NIH-funded Clinical Genome Resource (ClinGen) patient registry) may help make the recontact more routine and patient-focused, notifying patients when clinically significant new information becomes available that affects the interpretation of their genetic results (Kirkpatrick et al. 2015). A recent study showed that nearly $70 \%$ of reclassified variants were shared with GenomeConnect users (Savatt et al. 2021).

There are some limitations to this study. Although varied, our sample of HCPs is limited; other HCPs in other medical centers in Israel may express different views. In addition, professional administrators in the testing labs should be interviewed about their views of reclassification and recontact. Moreover, findings may be limited to the context of the Israeli health system which is socialized and universal and has a long-established genetics services as part of public health.

In conclusion, drawing on qualitative data from HCPs with vast experiences in referring to genomic testing, interpreting results, and following up patients, a clear gap exists between the growing uptake of genomic testing and the long-term use of the generated information. Moreover, we argue that recontacting should not be perceived as a singular "duty," but a "complex interplay of shifting discourses of responsibilities" (Doheny et al. 2018: 214). A structured system of storing genomic data, periodical reinterpretation, and re-contacting former patients (or their relatives in cases patients are deceased) should be generated to maximize the potential of genomic medicine. Until such endeavors are successful, we encourage educating patients and clinicians about apps such as GenomeConnect which provides genetic updates back to interested patients.

Acknowledgements We thank all the healthcare professionals who participated in the study.

Funding This is funded by Israel Science Foundation (grant no. 272/19) and the Binational US-Israel Science Foundation (grant no. 2018027). 
Data availability The datasets generated and/or analyzed during the current study are available from the corresponding author on reasonable request.

\section{Code availability N/A.}

\section{Declarations}

Ethics approval and consent to participate This study has been performed in accordance with the Declaration of Helsinki and has been approved by the Ethics Committee of Hadassah Medical Center \#0447-19-HMO. Informed consent to participate in the study has been obtained from participants.

Consent for publication N/A: This MS does not include identifying details, images, or videos relating to an individual person.

Conflict of interest The authors declare no competing interests.

\section{References}

Appelbaum PS, Parens E, Berger SM, Chung WK, Burke W (2020) Is there a duty to reinterpret genetic data? The ethical dimensions. Genet Med 22:633-639

Braun K, Könninger S (2018) Realizing responsibility: institutional routines, critical intervention, and the "big" questions in the controversy over non-invasive prenatal testing in Germany. New Genet Soc. https://doi.org/10.1080/14636778.2018.1495555

Carrieri D, Lucassen AM, Clarke AJ et al (2016) Recontact in clinical practice: a survey of clinical genetics services in the United Kingdom. Genet Med 18:876-881

Carrieri D, Dheensa S, Doheny S et al (2017) Recontacting in clinical practice: an investigation of the views of healthcare professionals and clinical scientists in the United Kingdom. Eur J Hum Genet 25(3):275-279. https://doi.org/10.1038/ejhg.2016.188

Carrieri D, Howard HC, Benjamin C et al (2019) European Society of Human Genetics. Recontacting patients in clinical genetics services: recommendations of the European Society of Human Genetics. Eur J Hum Genet. 27(2):169-182. https://doi.org/10. 1038/s41431-018-0285-1

Clayton EW, Appelbaum PS, Chung WK, Marchant GE, Roberts JL, Evans BJ (2021) Does the law require reinterpretation and return of revised genomic results? Genet Med 23(5):833-836. https:// doi.org/10.1038/s41436-020-01065-x

David KL et al (2019) Patient re-contact after revision of genomic test results: points to consider-a statement of the American College of Medical Genetics and Genomics (ACMG). Genet Med 21:769-771

Denzin NK, Lincoln Y (1994) Handbook of qualitative research. Sage, Thousand Oaks

Dheensa S, Carrieri D, Kelly S, Clarke A, Doheny S, Turnpenny P et al (2017) A 'joint venture' model of recontacting in clinical genomics: challenges for responsible implementation. Eur J Med Genet 60:403-409

Doheny S, Clarke A, Carrieri D et al (2018) Dimensions of responsibility in medical genetics: exploring the complexity of the "duty to recontact." New Genetics and Society 37(3):187-206. https://doi. org/10.1080/14636778.2018.1510309

Hart JT (1971) The inverse care law. Lancet 297:405-412

Kirkpatrick BE et al (2015) GenomeConnect: matchmaking between patients, clinical laboratories, and researchers to improve genomic knowledge. Hum Mutat 36:974-9782

Koifman A et al (2016) Chromosomal micro-array analysis-the Israeli Medical Association. https://cdn.doctorsonly.co.il/2016/04/Ne57_ ChromosomalMicroarray.pdf. Accessed 4 Oct 2021

Marchant G, Barnes M, Evans JP, LeRoy B, Wolf SM (2020) LawSeq Liability Task Force. From genetics to genomics: facing the liability implications in clinical care. J Law Med Ethics 48(1):11-43

Medendorp NM, Hillen MA, van Maarschalkerweerd PE, Aalfs CM, Ausems MG, Verhoef S et al (2020) 'We don't know for sure': discussion of uncertainty concerning multigene panel testing during initial cancer genetic consultations. Fam Cancer 19:65-76

Medendorp NM, Hillen MA, Visser LNC et al (2021) A randomized experimental study to test the effects of discussing uncertainty during cancer genetic counseling: different strategies, different outcomes? Eur J Hum Genet 29:789-799. https://doi.org/10.1038/ s41431-020-00799-1

Mersch J, Brown N, Pirzadeh-Miller S et al (2018) Prevalence of variant reclassification Following Hereditary Cancer Genetic Testing. JAMA 320(12):1266-1274. https://doi.org/10.1001/jama.2018. 13152

Millo T, Douiev L, Popper D, Shkedi-Rafid S (2021) Personalized prenatal genomic testing: couples' experience with choice regarding uncertain and adult-onset findings from chromosomal-microarray-analysis. Prenat Diagn 41:376-383. https://doi.org/10.1002/ pd.5856

Reuter C, Chun N, Pariani M, Hanson-Kahn A (2019) Understanding variants of uncertain significance in the era of multigene panels: through the eyes of the patient. J Genet Couns 28(4):878-886. https://doi.org/10.1002/jgc4.1130

Richards S, Aziz N, Bale S et al (2015) Standards and guidelines for the interpretation of sequence variants: a joint consensus recommendation of the American College of Medical Genetics and Genomics and the Association for Molecular Pathology. Genet Med 17(5):405-423

Otten E, Plantinga M, Birnie E, Verkerk MA, Lucassen AM, Ranchor $\mathrm{AV}$ et al (2015) Is there a duty to recontact in light of new genetic technologies? A systematic review of the literature. Genet Med 17:668-678

Savatt JM, Azzariti DR, Ledbetter DH et al (2021) Recontacting registry participants with genetic updates through GenomeConnect, the ClinGen patient registry. Genet Med. https://doi.org/10.1038/ s41436-021-01197-8

Shabani M, Thorogood A, Borry P (2016) Who should have access to genomic data and how should they be held accountable? Perspectives of Data Access Committee members and experts. Eur J Hum Genet 24(12):1671-1675

Publisher's note Springer Nature remains neutral with regard to jurisdictional claims in published maps and institutional affiliations. 